\title{
ASYMPTOTIC BEHAVIOR OF SOLUTIONS OF RETARDED DIFFERENTIAL EQUATIONS
}

\author{
G. LADAS, Y. G. SFICAS ${ }^{1}$ AND I. P. STAVROULAKIS ${ }^{2}$
}

ABSTRACT. In this paper we obtain sufficient conditions under which every solution of the retarded differential equation

$$
x^{\prime}(t)+p(t) x(t-\tau)=0, \quad t \geqslant t_{0},
$$

where $\tau$ is a nonnegative constant, and $p(t)>0$, is a continuous function, tends to zero as $t \rightarrow \infty$. Also, under milder conditions, we prove that every oscillatory solution of (1) tends to zero as $t \rightarrow \infty$. More precisely the following theorems have been established.

THEOREM 1. Assume that $\int_{t_{0}}^{\infty} p(t) d t=+\infty$ and $\lim _{t \rightarrow \infty} !_{t-\tau}^{t} p(s) d s<\pi / 2$ or $\lim \sup _{t \rightarrow \infty} \int_{t-\tau}^{t} p(s) d s<1$. Then every solution of (1) tends to zero as $t \rightarrow \infty$.

THEOREM 2. Assume that $\lim \sup _{t \rightarrow \infty} \int_{t-\tau}^{t} p(s) d s<1$. Then every oscillatory solution of (1) tends to zero as $t \rightarrow \infty$.

1. Introduction and preliminaries. In the present paper we obtain sufficient conditions under which every solution of the retarded differential equation

$$
x^{\prime}(t)+p(t) x(t-\tau)=0, \quad t \geqslant t_{0},
$$

where $\tau$ is a nonnegative constant and $p(t)$ is a continuous function tends to zero as $t \rightarrow \infty$. Also, under milder conditions, we prove that every oscillatory solution of (1) tends to zero as $t \rightarrow \infty$.

It is known, see for example [1,p. 330], that every solution of the retarded differential equation

$$
y^{\prime}(t)+p y(t-\tau)=0,
$$

where

$$
p>0 \text { and } 0 \leqslant p \tau<\pi / 2 \text {, }
$$

tends to zero as $t \rightarrow \infty$. This is because (3) implies that every root of the characteristic equation of (2) has negative real part.

More precisely, if $y\left(t ; t_{0}, \phi\right)$ denotes the solution of (2) satisfying the initial condition $y_{t_{0}}=\phi$ where $\phi \in C[-\tau, 0]$, then (see $[1$, p. 325]) there exist positive constants $M$ and $\gamma$ such that

$$
\left|y\left(t ; t_{0}, \phi\right)\right| \leqslant M\|\phi\| e^{-\gamma\left(t-t_{0}\right)}, \quad t \geqslant t_{0},
$$

Received by the editors July 30, 1982.

1980 Mathematics Subject Classification. Primary 34K25; Secondary 34K15.

Key words and phrases. Retarded differential equation, solutions tend to zero, oscillatory solution.

'On sabbatical leave from the Univertisy of Ioannina, Greece.

${ }^{2}$ On leave from the University of Ioannina, Greece. 
where

$$
\|\phi\|=\sup _{-\tau \leqslant s \leqslant 0}|\phi(s)| .
$$

Also, if $z\left(t ; t_{0}, 0\right)$ denotes the solution of

$$
z^{\prime}(t)+p z(t-\tau)=h(t), \quad t \geqslant t_{0},
$$

with zero initial function at $t_{0}$, that is, $z_{t_{1}}=0$, then (see [1, p. 336])

$$
\left|z\left(t ; t_{0}, 0\right)\right| \leqslant \frac{M}{\gamma} e^{(p+\gamma) \tau} \max _{t_{1},-s-t}|h(s)| .
$$

The following lemma is needed in the proof of Theorem 1 .

LemMa. Consider the retarded differential equation

$$
x^{\prime}(t)+x(t-\sigma(t))=0, \quad t=t_{0},
$$

where $0 \leqslant \sigma(t) \leqslant t$ is continuous and $\lim _{t, \%} \sigma(t)=\tau-\infty$ exists. Assume that

$$
\tau<\pi / 2 \text {. }
$$

Then every solution of (7) tends to zero as $t \rightarrow \infty$.

Proof. Let $x(t)$ be any solution of (7). Choose $t_{1} \geqslant t_{0}+2 \tau+2$ and also such that

$$
\sigma(t) \leqslant \tau+1, \quad t \geqslant t_{1}
$$

and

$$
(M / \gamma) e^{(1+\gamma) \tau}|\tau-\sigma(t)| \leqslant \frac{1}{2}, \quad t \geqslant t_{1}
$$

where the constants $M$ and $\gamma$ are as defined in (4) with $p=1$. Let $y(t)$ be the solution of

$$
y^{\prime}(t)+y(t-\tau)=0, \quad t \geqslant t_{1},
$$

with initial function $y_{t_{1}}=x_{t_{1}}$. Then, in view of (8), $y(t)$ tends to zero as $t \rightarrow \infty$. Set $z(t)=x(t)-y(t)$ and observe that $z(t)$ satisfies the equation

$$
z^{\prime}(t)+z(t-\tau)=x(t-\tau)-x(t-\sigma(t)), \quad t \geqslant t_{1},
$$

with zero initial function at $t_{1}$. Using (6), with $p=1$ and

$$
h(s)=x(s-\tau)-x(s-\sigma(s)),
$$

we find

$$
|z(t)| \leqslant \frac{M}{\gamma} e^{(1+\gamma) \tau} \max _{t_{1} \leqslant s \leqslant t}|x(s-\tau)-x(s-\sigma(s))| .
$$

Applying the mean value theorem and equation (7), we obtain

$$
|x(s-\tau)-x(s-\sigma(s))|=|\sigma(s)-\tau|\left|x^{\prime}(\xi)\right|=|\sigma(s)-\tau||x(\xi-\sigma(\xi))|,
$$

where $\xi$ is between $s-\tau$ and $s-\sigma(s)$. Then, setting

$$
B_{1}=\max _{t_{0} \leqslant s \leqslant t_{1}}|x(s)|,
$$


we find

$$
\begin{aligned}
& \max _{t_{1} \leqslant s \leqslant t}|x(s-\tau)-x(s-\sigma(s))| \leqslant \max _{t_{1} \leqslant s \leqslant t}|\sigma(s)-\tau| \cdot \max _{t_{0} \leqslant s \leqslant t}|x(s)| \\
& \leqslant \max _{t_{1} \leqslant s \leqslant t}|\sigma(s)-\tau|\left[B_{1}+\max _{t_{1} \leqslant s \leqslant t}|x(s)|\right] .
\end{aligned}
$$

Thus (12) implies

$$
|x(t)|-|y(t)| \leqslant \frac{M}{\gamma} e^{(1+\gamma) \tau} \max _{t_{1} \leqslant s \leqslant t}|\sigma(s)-\tau|\left[B_{1}+\max _{t_{1} \leqslant s \leqslant t}|x(s)|\right],
$$

and, in view of (10),

$$
|x(t)| \leqslant|y(t)|+\frac{1}{2}\left[B_{1}+\max _{t_{1} \leqslant s \leqslant t}|x(s)|\right] .
$$

Hence for every $T \geqslant t_{1}$ and for $t_{1} \leqslant t \leqslant T$ we have

$$
|x(t)| \leqslant|y(t)|+\frac{1}{2}\left[B_{1}+\max _{t_{1} \leqslant s \leqslant T}|x(s)|\right],
$$

and taking the maximum of both sides and rearranging terms we find

$$
\max _{t_{1} \leqslant s \leqslant T}|x(t)| \leqslant 2 \max _{t_{1} \leqslant s \leqslant T}|y(t)|+B_{1} .
$$

That is, $x(t)$ is a bounded function and so there exists a $B \geqslant B_{1}$ such that

$$
|x(t)| \leqslant B \text { for } t \geqslant t_{0} .
$$

Thus (13) yields

$$
|x(t)| \leqslant|y(t)|+2 B \frac{M}{\gamma} e^{(1+\gamma) \tau} \max _{t_{1} \leqslant s \leqslant t}|\sigma(s)-\tau|,
$$

which implies that

$$
\lim _{t \rightarrow \infty} x(t)=0
$$

The proof is complete.

Note. Professor Driver informed us that this lemma follows from known results in the stability theory of delay differential equations, namely, Theorem G [1, p. 394], by taking $F(t, \psi)=-\psi(-\sigma(t))$ and $h(t, \psi)=-\psi(-\sigma(t))+\psi(-\tau)$. However we presented our proof because it is simple and direct.

\section{Main results.}

THEOREM 1. Consider the retarded differential equation (1) where $\tau$ is a nonnegative constant and $p(t)>0$ is continuous. Assume that

$$
\int_{t_{0}}^{\infty} p(t) d t=+\infty
$$

$\lim _{t \rightarrow \infty} \int_{t-\tau}^{t} p(s) d s$ exists, and

$$
\lim _{t \rightarrow \infty} \int_{t-\tau}^{t} p(s) d s<\frac{\pi}{2} .
$$

Then every solution of (1) tends to zero as $t \rightarrow \infty$. 
ProOF. Set

$$
u=\sigma(t) \equiv \int_{t_{0}}^{t} p(s) d s, \quad t \geqslant t_{0},
$$

and observe that $\sigma^{-1}$ exists, $\lim _{t \rightarrow \infty} u(t)=\infty$, and

$$
\sigma(t-\tau)=\int_{t_{0}}^{t-\tau} p(s) d s=\int_{t_{0}}^{t} p(s) d s-\int_{t-\tau}^{t} p(s) d s=u-\int_{\sigma^{-1}(u)-\tau}^{\sigma^{-1}(u)} p(s) d s .
$$

That is,

$$
t-\tau=\sigma^{-1}\left(u-\int_{\sigma^{-1}(u)-\tau}^{\sigma^{-1}(u)} p(s) d s\right) .
$$

Then the transformation

$$
z(u)=x\left(\sigma^{-1}(u)\right)
$$

reduces (1) to

$$
z^{\prime}(u)+z\left(u-\int_{\sigma^{-1}(u)-\tau}^{\sigma^{-1}(u)} p(s) d s\right)=0 .
$$

In view of condition (15), the hypotheses of the Lemma are satisfied for equation (16) and therefore $\lim _{u \rightarrow \infty} z(u)=\lim _{t \rightarrow \infty} x(t)=0$. The proof of the theorem is complete.

The next theorem shows that we may relax the left-hand side of condition (15) by replacing limit by limit superior if we strengthen the right-hand side by replacing $\pi / 2$ by 1 .

THEOREM 2. Consider the retarded differential equation

$$
x^{\prime}(t)+p(t) x(t-\tau)=0
$$

where $\tau$ is a positive constant and $p(t)>0$ a continuous function. Assume that

$$
\limsup _{t \rightarrow \infty} \int_{t-\tau}^{t} p(s) d s<1 .
$$

Then every oscillatory solution of (1) tends to zero as $t \rightarrow \infty$.

Proof. Let $x(t)$ be an oscillatory solution of (1) which does not tend to zero as $t \rightarrow \infty$. Then there exists a sequence $t_{n}, n=1,2, \ldots$, of zeros of $x(t)$ with the property that $t_{n+1}-t_{n} \geqslant \tau$ and $x(t) \neq 0$ on $\left(t_{n}, t_{n+1}\right)$ for $n=1,2, \ldots$. Set

$$
s_{n}=\max _{t_{n} \leqslant t \leqslant t_{n+1}}|x(t)|, \quad n=1,2, \ldots
$$

It suffices to prove that the sequence $s_{n}$ tends to zero as $t \rightarrow \infty$. Observe that

$$
s_{n}=\left|x\left(\xi_{n}\right)\right|, \quad n=1,2, \ldots,
$$

for some $\xi_{n} \in\left(t_{n}, t_{n+1}\right)$ and that $x^{\prime}\left(\xi_{n}\right)=0$. Hence, from (1) $x\left(\xi_{n}-\tau\right)=0$. Set $\tau_{n}=\max \left\{t_{n}, \xi_{n}-\tau\right\}, n=1,2, \ldots$ Integrating (1) from $\tau_{n}$ to $\xi_{n}$ we obtain

$$
x\left(\xi_{n}\right)=-\int_{\tau_{n}}^{\xi_{n}} p(s) x(s-\tau) d s .
$$


Since $\tau_{n} \leqslant s \leqslant \xi_{n}$, it follows that $t_{n-1} \leqslant s-\tau \leqslant t_{n+1}$ and so, from (18),

$$
\left|x\left(\xi_{n}\right)\right| \leqslant \int_{\tau_{n}}^{\xi_{n}} p(s)|x(s-\tau)| d s \leqslant\left(\max _{t_{n-1} \leqslant t \leqslant t_{n+1}}|x(t)|\right) \int_{\tau_{n}}^{\tau_{n}+\tau} p(s) d s
$$

or

$$
s_{n} \leqslant\left(\max \left\{s_{n}, s_{n-1}\right\}\right) \int_{\tau_{n}}^{\tau_{n}+\tau} p(s) d s .
$$

In view of (17) it follows that for sufficiently large $n$, say $n \geqslant n_{0}$,

$$
s_{n} \leqslant s_{n-1} \int_{\tau_{n}}^{\tau_{n}+\tau} p(s) d s
$$

Now choose a number $\mu$ such that

$$
\limsup _{t \rightarrow \infty} \int_{t-\tau}^{t} p(s) d s<\mu<1 .
$$

Then for $N \geqslant n_{0}$ sufficiently large,

$$
\int_{\tau_{n}}^{\tau_{n}+\tau} p(s) d s \leqslant \mu<1, \quad n \geqslant N,
$$

and (19) yields

$$
s_{n} \leqslant \mu s_{n-1}, \quad n \geqslant N .
$$

This implies that

$$
\lim _{n \rightarrow \infty} s_{n}=0
$$

and the proof is complete.

If in addition to the hypotheses of Theorem 2 we assume that $\int_{t_{0}}^{\infty} p(s) d s=+\infty$, then it is easy to prove that every nonoscillatory solution of (1) tends to zero as $t \rightarrow \infty$. In fact, let $x(t)$ be a nonoscillatory solution of (1) which does not tend to zero as $t \rightarrow \infty$. Without loss of generality, we assume that for some $t_{1}>t_{0}, x(t)>0$. Thus for $t_{2}$ sufficiently large

$$
x(t)-x\left(t_{2}\right)+\int_{t_{2}}^{t} p(s) x(s-\tau) d s=0,
$$

and (because $x(t)$ decreases)

$$
x(t)-x\left(t_{2}\right)+x(t-\tau) \int_{t_{2}}^{t} p(s) d s \leqslant 0,
$$

which, as $t \rightarrow \infty$, leads to a contradiction.

The above observation together with Theorem 2 leads to the following result.

THEOREM 3. Consider the retarded differential equation (1) where $\tau$ is a nonnegative constant and $p(t)>0$ a continuous function. Assume that $\int_{t_{0}}^{\infty} p(t) d t=+\infty$ and

$$
\limsup _{t \rightarrow \infty} \int_{t-\tau}^{t} p(s) d s<1 .
$$

Then every solution of (1) tends to zero as $t \rightarrow \infty$. 
3. Examples. When $p(t)$ in equation (1) is a positive constant, the hypothesis (15) of Theorem 1 becomes $p \tau<\pi / 2$, which is the "best" condition for every solution of equation (2) to tend to zero as $t \rightarrow \infty$. Indeed, when $p \tau=\pi / 2$, as for example in

$$
y^{\prime}(t)+y(t-\pi / 2)=0,
$$

some solutions (e.g., $y(t)=\sin t$ or $\cos t$ ) do not tend to zero as $t \rightarrow \infty$.

Observe that the hypothesis (17) of Theorem 2 is not satisfied by equation (20) and therefore it is not surprising that the oscillatory solutions $y(t)=\sin t$ or $\cos t$ of (20) do not tend to zero as $t \rightarrow \infty$.

REMARK 1. Note that Theorem 2 describes the asymptotic behaviour of the oscillatory solutions only. Now the question is the following: Are there cases where all solutions of (1) oscillate and therefore Theorem 2 describes the asymptotic behavior of all solutions as Theorem 1? The answer to this question is yes and such conditions are described in [2], namely

$$
p(t)>0, \quad \liminf _{t \rightarrow \infty} \int_{t-\tau / 2}^{t} p(s) d s>0,
$$

and

$$
\liminf _{t \rightarrow \infty} \int_{t-\tau}^{t} p(s) d s>\frac{1}{e} .
$$

EXAMPLE 1. Consider the retarded differential equation

$$
x^{\prime}(t)+p(2+\cos t) x(t-2 \pi)=0, \quad t \geqslant 0,
$$

where $0<p<\frac{1}{8}$.

Observe that for $p(t)=p(2+\cos t)$,

$$
\int_{0}^{\infty} p(t) d t=+\infty \text { and } \int_{t-2 \pi}^{t} p(s) d s=4 p \pi<\frac{\pi}{2}
$$

that is the hypotheses of Theorem 1 are satisfied. Therefore every solution of this equation tends to zero as $t \rightarrow \infty$.

EXAMPLE 2. Consider the retarded differential equation

$$
x^{\prime}(t)+p(2+\cos t) x(t-\pi)=0, \quad t \geqslant 0,
$$

where $0<p<1 / 2(\pi+1)$.

Observe that for $p(t)=p(2+\cos t)$

$$
\int_{0}^{\infty} p(t) d t=+\infty \text { and } \int_{t-\pi}^{t} p(s) d s=\int_{t-\pi}^{t} p(2+\cos s) d s=2 p(\pi+\sin t) .
$$

Moreover,

$$
\limsup _{t \rightarrow \infty} \int_{t-\pi}^{t} p(s) d s=2 p(\pi+1)<1
$$

and, therefore, the hypotheses of Theorem 3 are satisfied. Thus every solution of this equation tends to zero as $t \rightarrow \infty$. 
EXAMPLE 3. If $\tau<\pi / 2$ then, according to the Lemma, every solution of each of the following retarded differential equations

$$
x^{\prime}(t)+x(t-\tau-1 / t)=0 \text { and } x^{\prime}(t)+x\left(t-\tau-e^{-t}\right)=0
$$

tends to zero as $t \rightarrow \infty$.

\section{REFERENCES}

1. R. D. Driver, Ordinary and delay differential equations, Springer-Verlag, Berlin and New York, 1977.

2. G. Ladas, Sharp conditions for oscillations caused by delays, Applicable Anal. 9 (1979), 93-98.

Department of MAThematics, University of Rhode ISLAND, Kingston, Rhode Island 02881 